\title{
Per- and polyfluoroalkyl substances in Chinese and German river water - Point source- and country-specific fingerprints including unknown precursors ${ }^{\text {मै }}$
}

\author{
Hanna Joerss ${ }^{\text {a, b }}$, Thekla-Regine Schramm a , Linting Sun ${ }^{\text {c, d }}$, Chao Guo c, d, \\ Jianhui Tang ${ }^{c, e, ~}{ }^{*}$, Ralf Ebinghaus ${ }^{\text {a }}$ \\ ${ }^{a}$ Helmholtz-Zentrum Geesthacht, Institute of Coastal Research, 21502, Geesthacht, Germany \\ ${ }^{\mathrm{b}}$ Universität Hamburg, Institute of Inorganic and Applied Chemistry, 20146, Hamburg, Germany \\ c CAS Key Laboratory of Coastal Environmental Processes and Ecological Remediation, Shandong Key Laboratory of Coastal Environmental Processes, Yantai \\ Institute of Coastal Zone Research, Chinese Academy of Sciences (CAS), Yantai, 264003, China \\ d University of Chinese Academy of Sciences, Beijing, 100049, China \\ e Center for Ocean Mega-Science, Chinese Academy of Sciences, Qingdao, 266071, China
}

\section{A R T I C L E I N F O}

\section{Article history:}

Received 9 June 2020

Received in revised form 26 August 2020

Accepted 27 August 2020

Available online 31 August 2020

\section{Keywords:}

Emerging contaminants

PFASs

Surface water

LC-MS/MS

Isomer profiling

Total oxidizable precursor assay

\begin{abstract}
A B S T R A C T
This study aimed at comparing source-specific fingerprints of per- and polyfluoroalkyl substances (PFASs) in river water from China and Germany, selected as countries with different histories of PFAS production. Samples were collected from up- and downstream of seven suspected point sources in autumn 2018. Amongst the 29 analyzed legacy and emerging PFASs, 24 were detected, with a sum ranging from $2.7 \mathrm{ng} / \mathrm{L}$ (Alz River) to 420,000 ng/L (Xiaoqing River). While mass flow estimates for the Xiaoqing River and Yangtze River (mean: 20 and 43 t/y, respectively) indicated ongoing high emissions of the legacy compound PFOA in China, its ether-based replacements HFPO-DA and DONA showed the highest contribution downstream of a German fluoropolymer manufacturing site ( $50 \%$ and $40 \%$ of $\Sigma$ PFASs measured, respectively). In river water impacted by manufacturing sites for pharmaceutical and pesticide intermediates, the short-chain compound PFBS was the most prevalent substance in both countries. The German Ruhr River, receiving discharges from the electroplating industry, was characterized by the PFOS replacement 6:2 FTSA. Isomer profiling revealed a higher proportion of branched isomers in the Chinese Xi River and Xiaoqing River than in other rivers. This points to different synthesis routes and underlines the importance of differentiating between linear and branched isomers in risks assessments. Upon oxidative conversion in the total oxidizable precursor (TOP) assay, the increase of the short-chain compound PFBA was higher in German samples than in Chinese samples $(88 \pm 30 \%$ versus $12 \pm 14 \%)$, suggesting the presence of a higher proportion of unknown precursors to PFBA in the German environment. Amongst the ether-based replacements, DONA and 6:2 Cl-PFESA were fully or partially degraded to non-targeted oxidation products, whereas HFPO-DA showed no degradation. This indicates that the inclusion of ether-based PFASs and their oxidation products in the TOP assay can help in capturing a larger amount of the unknown PFAS fraction.
\end{abstract}

(ㄷ) 2020 Elsevier Ltd. All rights reserved.

\footnotetext{
* This paper has been recommended for acceptance by Charles Wong.

* Corresponding author. CAS Key Laboratory of Coastal Environmental Processes and Ecological Remediation; Shandong Key Laboratory of Coastal Environmental Processes, Yantai Institute of Coastal Zone Research, Chinese Academy of Sciences (CAS), Yantai, 264003, China.

E-mail addresses: hanna.joerss@hzg.de (H. Joerss), thekla-regine.schramm@ web.de (T.-R. Schramm), Itsun@yic.ac.cn (L. Sun), cguo@yic.ac.cn (C. Guo), jhtang@yic.ac.cn (J. Tang), ralf.ebinghaus@hzg.de (R. Ebinghaus).
}

\section{Introduction}

Per- and polyfluoroalkyl substances (PFASs) are a group of anthropogenic chemicals that have been used for over 70 years due to their high thermal and chemical stability along with their amphiphilic nature (Kissa, 2001). There are currently 6330 PFASrelated Chemical Abstract Service (CAS) registry numbers included in the "PFAS Master List", which was compiled by the United States Environmental Protection Agency (US EPA) based on 
the "New Comprehensive Database of PFASs" (OECD, 2018) and other publicly available lists (US EPA, 2020). The chemicals are used in a wide range of industrial applications and consumer products, such as fluoropolymer manufacture, fire-fighting foams, metal plating, and surface treatment agents (Buck et al., 2011). Among the large number of PFASs, research has focused on long-chain perfluoroalkyl carboxylic acids (PFCAs, $\mathrm{C}_{n} \mathrm{~F}_{2 n+1} \mathrm{COOH}, n \geq 7$ ) and perfluoroalkyl sulfonic acids (PFSAs, $\mathrm{C}_{n} \mathrm{~F}_{2 n+1} \mathrm{SO}_{3} \mathrm{H}, n \geq 6$ ), leading to their recognition as global contaminants of high concern. Along with their gradual phase-out and regulations in North America, Europe, and Japan, an industrial transition has taken place. Production of long-chain PFCAs and PFSAs has been shifted to countries with less stringent regulations, especially China and other Asian countries (Wang et al., 2014), and production has moved towards replacement compounds (Wang et al., 2013b). The common practice to replace one phased-out compound by multiple others is one reason for an increasingly large and diverse number of PFASs (Wang et al., 2017). Potential adverse properties, environmental occurrence and fate of replacements are often largely unknown when the compounds are introduced into the market. By means of conventional compound-specific analytical methods, only a small fraction of the PFASs on the global market can be determined, indicating that environmental and human exposure to PFASs may be underestimated. To address this concern, targeted analytical methods have been complemented with sum parameters (McDonough et al., 2019) and high-resolution mass spectrometry (HRMS)-based approaches (Liu et al., 2019) to characterize the unknown pool of PFASs. Sum parameters include the Total Oxidizable Precursor (TOP) assay, which provides an estimate of the total precursors in a sample that oxidize to (selected) targeted PFASs (Houtz and Sedlak, 2012). Application of the TOP assay to river water samples from Japan (Ye et al., 2014) and France (Boiteux et al., 2017) has revealed a significant proportion of unidentified precursors, albeit varying in amount. However, the aforementioned studies have not considered replacement compounds, such as perand polyfluoroalkyl ether carboxylic and sulfonic acids (PFECAs and PFESAs), as terminal products or precursors in the TOP assay. A recent study highlighted that this could result in missing a fraction of the total PFAS amount (Zhang et al., 2019).

Studies investigating the impact of industrial point sources on the environment typically focus on one study site, one geographical region, and one type of source (Jin et al., 2015; Shi et al., 2015; Chen et al., 2018). Due to differing analytical scopes in target analysis, especially regarding emerging PFASs, and non-standardized bulk and HRMS-based approaches, there is often a lack of comparability across studies. Consequently, a comparison of various types of point sources from regions with a different history of PFAS production using one set of analytical methods can provide a more comprehensive picture.

In this study, river water samples were collected at 58 locations, up- and downstream of suspected point sources at three sites in China and four sites in Germany. The sources covered major areas of PFAS production and application, including fluoropolymer production, manufacturing sites for pharmaceutical and pesticide intermediates, and the electroplating industry. The aim was to characterize the different study sites by PFAS "fingerprints", based on the hypothesis that the fingerprints depend on the type of point source and on the history of PFAS production in the two countries. More specifically, the objectives were i) to investigate occurrence and composition profiles of 29 legacy and emerging PFASs by using target analysis, ii) to further characterize source fingerprints based on isomer profiling and principal component analysis (PCA), iii) to evaluate the significance of unknown precursors relative to targeted PFASs by applying the TOP assay with an expanded list of target analytes, including replacement compounds, and iv) to estimate riverine PFAS mass flows.

\section{Materials and methods}

\subsection{Study sites}

River water samples were collected at seven sites, which had been previously reported to be impacted by industrial point sources (Figs. S1-S3; Table S1). Site XQ was located in the Chinese Xiaoqing River basin (Huantai, Shandong Province), receiving discharge from one of the largest Asian fluoropolymer production sites (Fig. S3). The chemical park's annual polytetrafluoroethylene (PTFE) production capacity was rapidly expanded from $3 \mathrm{kt}$ in 2002 to $30 \mathrm{kt}$ in 2009 (Wang et al., 2014). In 2015, its capacity was $44.3 \mathrm{kt}$ of PTFE, $13 \mathrm{kt}$ of vinylidene fluoride (VDF), $10 \mathrm{kt}$ of hexafluoropropylene (HFP), $10 \mathrm{kt}$ of fluoroelastomers (FKM), and $8.4 \mathrm{kt}$ of polyvinylidene fluoride (PVDF) (Song et al., 2018). Site FX was situated at the Xi River (Fuxin, Liaoning Province, Daling River Basin), where two fluorochemical industrial parks have been built due to the local abundance of fluorite (Bao et al., 2011). Its main products include short-chain PFAAs and other fluorine-containing pharmaceutical and pesticide intermediates, as well as fluorotelomers (Chen et al., 2018). Site YZ is located at the Yangtze River in Changshu, Jiangsu Province, and in close proximity to the "Advanced Materials Industrial Park (AMIP)" with more than 15 domestic and overseas fluorochemical companies (Jin et al., 2015). Various plants for different operations have been built since 1999, e.g., for production of PTFE, PVDF, and fluoroelastomers.

In Germany, samples were collected from the Alz River (site AZ), up- and downstream of a chemical park for production of fluoropolymers and fluoromonomers (Fig. S2). Here, salts of PFOA were used as a processing aid until 2008, before being replaced by the ether-based compound ammonium 2,2,3-trifluoro-3-[1,1,2,2,3,3hexafluoro-3-(trifluoromethoxy)propoxy]propionate (ADONA) (Fromme et al., 2017). The three additional German sites were located along the Rhine River and its tributaries. Site MA is situated at the Main River tributary, before discharging into the Rhine River and close to Frankfurt Airport as well as a chemical park with more than 90 companies. Amongst others, pesticide formulations containing perfluoroalkyl phosphinic acids (PFPiAs) and perfluoroalkyl phosphonic acids (PFPAs) are produced by a large agricultural chemical company operating here (Wang et al., 2016b). Approximately $200 \mathrm{~km}$ downstream of the Main River mouth, the largest chemical park in Germany is situated at the Rhine River, mainly dedicated to the production of pharmaceuticals and pesticides (site $\mathrm{RH}$ ). In addition, samples were collected from the Ruhr River (site RU). Historically characterized by coal mining, iron, and steel production since the 19th century (Bode, 1998), the Ruhr Area is still a center of metal plating. The River Rhine tributary Lahn was selected as reference site because it had been previously described as a river with little industrial influence and background PFAS concentrations (Skutlarek et al., 2006; Möller et al., 2010).

\subsection{Sample collection}

Sampling was performed from pontoons, bridges, stone piers or the riverside in September 2018 (Germany) and November 2018 (China). Water was collected at a depth $0.5 \mathrm{~m}$ from the surface at six to ten sampling locations per site, up- and downstream of the potential source (Figs. S2 and S3). In total, samples for target analysis were collected at 58 stations in 1-L polypropylene (PP) bottles. At 31 of the stations, two aliquots of each sample were filled into $125 \mathrm{~mL}$ high density polyethylene (HDPE) bottles for application of the TOP assay. Samples were stored at $4{ }^{\circ} \mathrm{C}$ and processed in the laboratory within four weeks after sampling. Water temperature, 
salinity, and $\mathrm{pH}$ were measured onsite using a portable measuring device. The sampling coordinates and the results for the physicochemical parameters are given in Tables S2a and S2b.

\subsection{Target analytes and chemicals}

The analytical method included 29 target analytes from eight structural classes: 11 PFCAs $\left(C_{4}\right.$ to $\left.C_{14}\right)$, five PFSAs $\left(C_{4}, C_{6}, C_{7}, C_{8}, C_{10}\right)$, the cyclic PFAS PFECHS, four PFECAs and PFESAs (HFPO-DA, ADONA; 6:2 and 8:2 Cl-PFESA), two PFPiAs (6:6 PFPiA, 6:8 PFPiA), three fluorotelomer sulfonic acids (4:2 FTSA, 6:2 FTSA, 8:2 FTSA), and three sulfonamide-containing precursors (FOSA, $N$-EtFOSE, $N$ EtFOSAA). A total of 15 internal standards were used, which included seven isotopically labelled PFCAs $\left({ }^{13} \mathrm{C}_{4}\right.$-PFBA, ${ }^{13} \mathrm{C}_{2}$-PFHxA, ${ }^{13} \mathrm{C}_{4}$-PFOA, ${ }^{13} \mathrm{C}_{5}$-PFNA, ${ }^{13} \mathrm{C}_{2}$-PFDA, ${ }^{13} \mathrm{C}_{2}$-PFUnDA, ${ }^{13} \mathrm{C}_{2}$-PFDoDA), three PFSAs $\left({ }^{13} \mathrm{C}_{3}\right.$-PFBS, ${ }^{18} \mathrm{O}_{2}$-PFHXS, ${ }^{13} \mathrm{C}_{4}$-PFOS), one PFECA $\left({ }^{13} C_{3}\right.$-HFPO-DA), two FTSAs $\left({ }^{13} C_{2}-4: 2\right.$ FTSA, ${ }^{13} C_{2}-8: 2$ FTSA), and two sulfonamide-containing precursors $\left({ }^{13} \mathrm{C}_{8}\right.$-FOSA, d9- $N$-EtFOSE). ${ }^{13} \mathrm{C}_{8}$-PFOA was used as the injection standard. CAS numbers of the target analytes and information on the analytical standards are provided in Tables S3 and S4. Additional chemicals used for sample analysis are listed in Table S5.

\subsection{Sample pretreatment and extraction}

Glass microfiber filters (Whatman, grade: GF/F, pore size: $0.7 \mu \mathrm{m}$, diameter: $47 \mathrm{~mm}$, GE Healthcare, USA) were used for filtration of the samples after baking the filters at $450{ }^{\circ} \mathrm{C}$ in a Muffle furnace overnight. Based on concentrations reported in previous studies (low ng/L to high $\mu \mathrm{g} / \mathrm{L}$ range) (Heydebreck et al., 2015; Jin et al., 2015; Chen et al., 2018) and the linear range of the instrument, samples from the different sites were grouped in four categories (Table S6). Accordingly, $1 \mathrm{~L}$ river water, $500 \mathrm{~mL}, 100 \mathrm{~mL}$, or $1 \mathrm{~mL}$ diluted in $250 \mathrm{~mL}$ ultrapure water were used for target analysis. Solid phase extraction (SPE) was performed as described previously (Joerss et al., 2019). Briefly, samples were spiked with internal standards (5 ng each) and loaded onto preconditioned SPE cartridges (Oasis WAX, $6 \mathrm{cc}, 500 \mathrm{mg}$ sorbent, $60 \mu \mathrm{m}$ particle size, Waters, USA). After a washing step with $15 \mathrm{~mL}$ of an 80:20 (v/v) water/methanol solution, the cartridges were dried under vacuum. Extraction of the German samples took place at HelmholtzZentrum Geesthacht (HZG), whereas Chinese samples were processed at the Yantai Institute of Coastal Zone Research (YIC). To ensure comparability of sample treatment, the samples were extracted by the same person using the same materials and chemicals in both countries. The dried cartridges were shipped from China to Germany and all further analytical steps were performed at HZG (Section S1). PFASs were analyzed by liquid chromatography coupled with tandem mass spectrometry (LC-MS/MS), of which detailed information is given in Tables S7-S9. Quantification of PFASs, including linear ( $n-)$ and the sum of branched $(b r-)$ isomers of target analytes, is explained in Section S2.

The TOP assay was performed according to Houtz and Sedlak (2012). One of the two $125 \mathrm{~mL}$ sample aliquots was amended with $2 \mathrm{~g}$ potassium persulfate $(60 \mathrm{mM})$ and $1.9 \mathrm{~mL}$ of $10 \mathrm{~N}$ sodium hydroxide solution $(150 \mathrm{mM})$. The samples were placed in a temperature-controlled water bath at $85{ }^{\circ} \mathrm{C}$ for $6 \mathrm{~h}$. After cooling them down in an ice bath, the $\mathrm{pH}$ of the samples was adjusted to a value between 6 and 8 . The oxidized aliquot and a second untreated aliquot were spiked with internal standards (1.5 ng each) and processed using the target analysis method.

\subsection{Quality assurance and quality control for target analysis}

A 14-point calibration curve ranging from $0 \mathrm{pg} / \mu \mathrm{L}$ to $100 \mathrm{pg} / \mu \mathrm{L}$ was generated and measured before and after each sample batch (Section S2). Due to prior categorization of the samples and extraction of different sample volumes, measured concentrations were mostly within the linear range of the instrument. If the linear range was exceeded, the extract was diluted with the sample solvent and measured again. In some cases, wherein concentrations were still above the calibration range, results must be considered as semiquantitative (marked in Tables S17 and S18).

Although glass fiber filters were shown to result in considerably lower sorption of PFCAs, PFSAs and FTSAs than other filter types, their use may still result in significant sorption of PFPiAs and in the underestimation of PFPiA concentrations (Chandramouli et al., 2015). Matrix spike recovery tests for PFASs in Elbe river water at a spiking level of $3 \mathrm{ng} / \mathrm{L}$ resulted in relative recoveries from $78 \pm 1 \%$ to $109 \pm 5 \%$, except for $6: 8$ PFPiA with a recovery of $60 \pm 3 \%$ (Table S10). Absolute recoveries of internal standards are provided in Table S11.

Results for target analytes that were detected in the procedural laboratory blank samples were corrected by subtracting the average PFAS concentration in the blank samples ( $\mathrm{pg} / \mathrm{L}$ range) from the concentrations in the samples. Mean blank values added to 3 or 10 times the standard deviation were set as method detection limits (MDLs) and method quantification limits (MQLs) for compounds present in the blank samples. For the remaining target analytes, the calculation of the MDLs and MQLs was based on a signal-to-noise ratio of 3 or 10, derived from low-level or spiked samples. As samples from German and Chinese rivers were processed in different laboratories, campaign-specific MDLs and MQLs were calculated. MQLs ranged from $0.017 \mathrm{ng} / \mathrm{L}$ (FOSA) to $0.33 \mathrm{ng} / \mathrm{L}$ (PFBA) in 1-L water samples. Lower sample volumes resulted in correspondingly higher MQLs (Table S12). Relative standard deviations of quantifiable compounds in triplicate samples were below $20 \%$.

\subsection{Quality assurance and quality control for the TOP assay}

To validate the TOP assay, oxidation tests with model substances were performed, including $n: 2$ fluorotelomer sulfonic acids (4:2 FTSA and 6:2 FTSA), sulfonamide-containing precursors ( $N$-EtFOSAA, N-EtFOSE), and ether-based replacement compounds (ADONA; HFPO-DA and 6:2 Cl-PFESA). The single compounds were added to ultrapure water at a spiking level of $15 \mathrm{ng} / \mathrm{L}(n=3)$ and the TOP assay was performed as described in section 2.4 . The results of the oxidation tests are discussed in section 3.4.

Absolute recoveries of internal standards were lower in oxidized sample aliquots compared to unoxidized aliquots (Table S11). This difference has also been reported by Janda (2019). A possible explanation is that the sulfate anions in the oxidized aliquots, generated upon oxidation of persulfate, may compete with the anionic target analytes for ion-exchange sites of the SPE sorbent. In addition, concentrations of particular target analytes $\left(C_{4}-C_{8}\right.$ PFCAs, PFOS) were higher in oxidized procedural blanks than in unoxidized procedural blanks. Consequently, MDLs and MQLs were calculated separately for oxidized and unoxidized samples. For the German sampling campaign, MQLs were in the range of $0.50 \mathrm{ng} / \mathrm{L}$ (PFUnDA) to $2.5 \mathrm{ng} / \mathrm{L}$ (PFOS) for unoxidized samples and of $0.50 \mathrm{ng} /$ $\mathrm{L}$ (PFUnDA) to $7.9 \mathrm{ng} / \mathrm{L}$ (PFOA) for oxidized samples (Table S14). Relative standard deviations of quantifiable compounds in both aliquots of triplicate samples were below $20 \%$.

\subsection{Data analysis}

PCA was performed with OriginPro, 2018 (version 9.5) on the proportions of single PFAS concentrations to the sum of PFASs to compare the different source patterns. Eigenvalue decomposition 
was performed on the correlation matrix of the dataset. Only PFASs with a detection frequency $>40 \%$ were included. Measured values between MDL and MQL were used unaltered for the calculations, results $<$ MDL were considered as $\sqrt{ } 2 / 2$. MDL.

PFAS mass flows in the respective rivers [t/year] were calculated by multiplying the measured concentration [ng/L] with the mean annual water flow of the river [L/year] and $10^{-15}$ as the conversion factor from ng to t. Water flow data was compiled from public sources for the gauging stations closest to the respective sampling sites (Table S15).

\section{Results and discussion}

\subsection{PFAS concentrations and composition patterns}

Amongst the 29 target analytes, all except PFTeDA, PFDS, 6:8 PFPiA, 8:2 Cl-PFESA and $N$-EtFOSE were detected in the Chinese and/or German river water samples. The sum of the detected compounds ranged from $2.7 \mathrm{ng} / \mathrm{L}$ in the German Alz River upstream of the point source (sample AZ_002) to 420,000 ng/L in the Chinese Xiaoqing River Basin downstream of the point source (sample XQ_007). Results for individual samples are provided in Tables S16-S19.

HFPO-DA, used to substitute PFOA in fluoropolymer manufacture (Wang et al., 2013b), was detected in $98 \%$ of all samples. This underlines the widespread use and ubiquitous presence of this compound. In contrast, 6:2 Cl-PFESA only occurred in China, having a detection frequency of $82 \%$. Presumably, this is related to the production and use of $\mathrm{F}-53 \mathrm{~B}$, which contains the potassium salt of 6:2 Cl-PFESA as a major compound and has been applied by Chinese manufacturers as alternative to PFOS salts in metal plating since the 1970s (Wang et al., 2013a). The compounds ADONA, PFECHS, and 6:6 PFPiA were predominantly detected in German samples. However, lower detection frequencies in the Chinese samples can result from higher MDLs (Table S12). The trimer acid of hexafluoropropylene oxide (HFPO-TrA), which has been reported to be present in river water before (Pan et al., 2018), was not included in our study. Amongst the PFCAs, PFSAs, and their precursors, detection frequencies of $\geq 90 \%$ in samples from both countries were observed for $\mathrm{C}_{4}-\mathrm{C}_{9}$ PFCAs, PFBS, $n$-PFHxS, and $n$-PFOS.

The comparison of PFAS concentrations and patterns before and after the suspected point sources clearly indicated the impact of the source at five of the seven sites; this is exemplified for sites XQ and AZ in Fig. 1. The exceptions were the sites at the Yangtze River (site $Y Z$ ) and the Ruhr River (site RU), where no significant changes in the PFAS concentration and pattern were observed. For site YZ, a strong dilution due to the large river size (mean annual discharge $27,400 \mathrm{~m}^{3} / \mathrm{s}$ ) in combination with an already high PFAS load due to upstream sources (Pan et al., 2018) may be an explanation. For site $\mathrm{RU}$, the results indicate that major sources are further upstream than suspected.

PFAS composition patterns of the different point sources are compared in Fig. 2. The well-known legacy compound PFOA was the most prevalent substance at the Chinese fluoropolymer manufacturing site, $\mathrm{XQ}$, contributing approximately $90 \%$ to $\Sigma$ PFASs. In contrast, its replacement compounds HFPO-DA and ADONA were the predominant PFASs found near the German fluoropolymer production facility at site $\mathrm{AZ}$, contributing approximately $50 \%$ and $40 \%$ to $\Sigma$ PFASs, respectively. This difference between Chinese and German fluoropolymer production sites reflects the geographical shift of production with a phase-out of long-chain PFCAs in Europe and a limited phase-out in Asian countries (Wang et al., 2014). The short-chain compound PFBS was the predominant substance downstream of the point sources at the Xi River in China (site FX) and the Rhine River in Germany (site RH), with a share of $70 \pm 6 \%$, or $66 \pm 8 \%$, respectively. At these sites, pharmaceutical and pesticide intermediates are produced or applied, indicating the importance of short-chain PFASs or their precursors in this sector. This is reinforced by a global market report, according to which the second and third largest application of PFBS in 2015 was its use as an intermediate in the pharmaceutical industry (4.4 t) and as an insecticide or for manufacture of insecticides $(1.4 \mathrm{t}) .19 \mathrm{t}$ of PFBS were used as surfactants or for the manufacture of surfactants (Norwegian Environment Agency, 2017). Typically, PFBS, its salts and other PFBS-related chemicals are synthesized based on perfluorobutane sulfonyl fluoride (PBSF), of which pesticide production was the second largest application globally in 2015 (88 t) (Norwegian Environment Agency, 2017). In comparison to the Lahn River as a River Rhine tributary with little industrial influence (site REF), the Ruhr River was characterized by a higher proportion of $6: 2$ FTSA ( $1.1 \%$ of total PFASs vs. $14 \pm 3 \%)$. This can be related to the hard chrome plating shops located in the highly industrialized Ruhr catchment, where salts of 6:2 FTSA are typically used to replace PFOS salts as mist suppressants (Wang et al., 2013b). The Main River (site MA) showed a similar profile compared to site REF, albeit with a marginally higher proportion of PFHxS, PFECHS, HFPO-DA, and 6:6 PFPiA. This may be attributed to the airport or chemical park located here. The Yangtze River (site YZ) was characterized by a large contribution of PFOA ( $40 \pm 11 \%)$ and PFHxA (39 $\pm 12 \%)$.

\subsection{Source characterization by principal component analysis}

PCA revealed three distinct groupings of PFASs, explaining $72 \%$ of the variability in river water concentrations (Fig. 3). Different statistical treatments of values below the MDL (zero, $\sqrt{ } 2 / 2 * \mathrm{MDL}$ and MDL) did not change the general outcome of the PCA. Detailed loadings are provided in Fig. S5.

The first component explained $41 \%$ of the variability and included legacy long-chain PFASs other than PFOA (PFNA, PFDA, $\Sigma$ PFOS, $\Sigma$ PFHxS, and $\Sigma$ FOSA), as well as the short-chain PFCAs PFPeA and $\Sigma$ PFHpA. This component separated the German sites RU, MA and RH (upstream of the point source) from the other sites, corresponding to the mixed patterns with comparable contribution of various PFASs shown in Fig. 2. The second component explained $16 \%$ of the variability and was dominated by the short-chain homologues PFBS and PFBA (with loadings of -0.52 and -0.48 , respectively) and the ether-based replacements HFPO-DA and ADONA (with loadings of 0.47 each). It had high scores for the $\mathrm{Xi}$ River samples (site FX) with PFBS and PFBA as dominating compounds and the Alz River samples (site AZ) with a high contribution of HFPO-DA and ADONA. The third component explained $14 \%$ of the variability and had high negative loadings of PFCAs ( $\Sigma$ PFOA and PFHxA) and the PFOS alternative 6:2 Cl-PFESA. Based on this component, the Xiaoqing River Basin and Yangtze River sites were distinguishable from other rivers.

The PCA plot shows a clear distinction between the samples taken before and after the respective point source for sites $\mathrm{AZ}, \mathrm{XQ}$ and $\mathrm{RH}$. The closer the upstream samples were taken to the source, the smaller was the variability in PFAS profiles in comparison to the downstream samples, as shown for site $\mathrm{XQ}$ and site $\mathrm{AZ}$ in Fig. 3. Upstream samples taken close to the source might be influenced by stack emissions or other ways of PFAS discharge.

\subsection{Source characterization by isomer profiling}

The contribution of branched isomers to the sum of the respective compounds revealed differences between rivers and countries (Table S20). In the German samples, the percentage of the sum of branched PFOS isomers (br-PFOS) was $19 \pm 7 \%$ (Fig. 4A). This is in accordance with a previous study in Europe, reporting a 

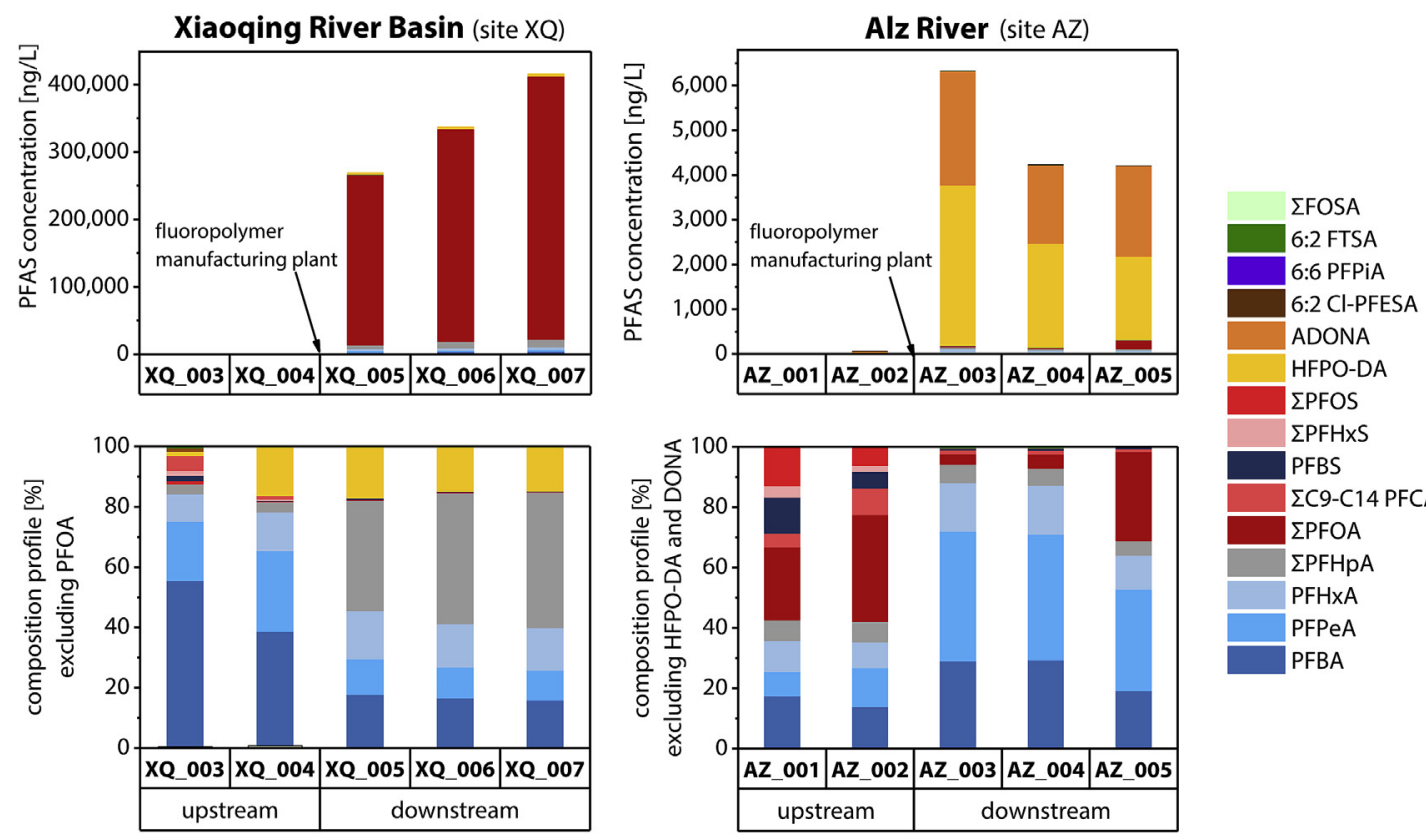

HFPO-DA

$\Sigma P F O S$

$\Sigma \mathrm{PFH}$ XS

PFBS

$\Sigma$ C9-C14 PFCAs

$\Sigma P F O A$

$\Sigma$ PFHpA

PFHxA

PFPeA

PFBA

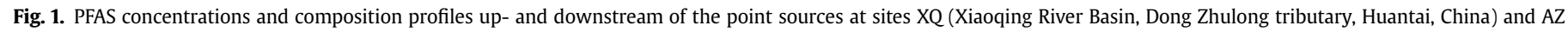
(Alz River, Burgkirchen, Germany). The composition profiles do not include the most dominant compounds (PFOA at site XQ and HFPO-DA and ADONA at site AZ).

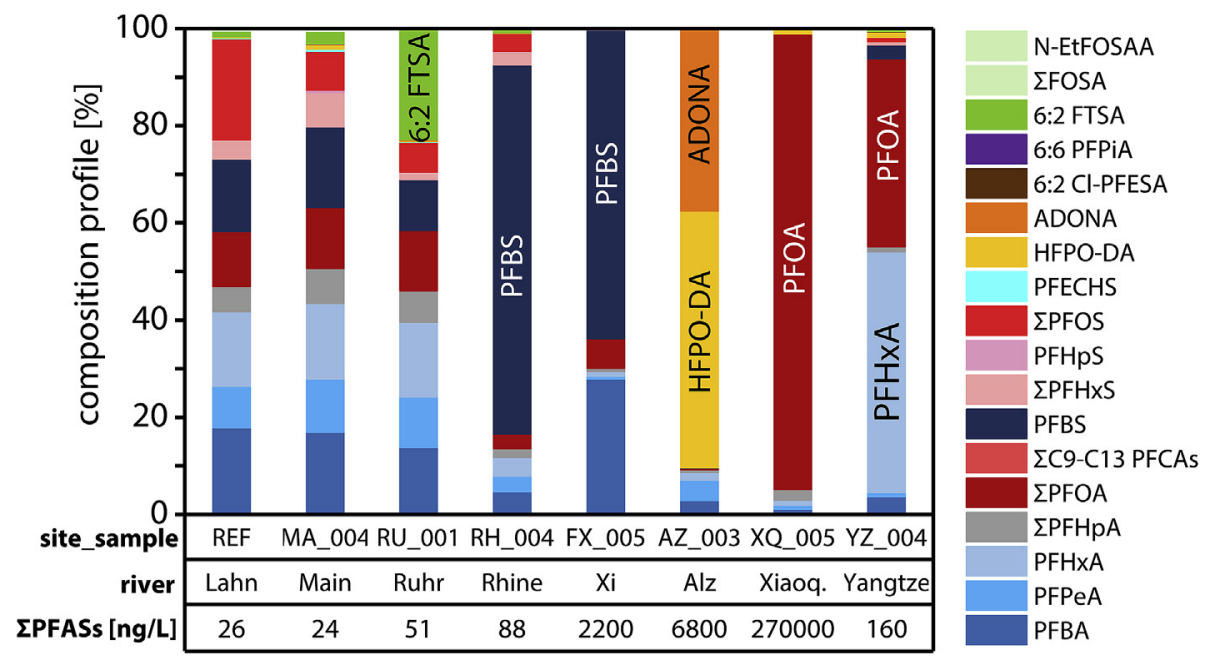

Fig. 2. Contributions of individual PFASs to $\mathrm{PPFASs}$ in selected samples, downstream of the suspected point sources in the investigated rivers.

contribution of $20 \%$ br-PFOS in Swedish surface waters $(n=285)$ (Gobelius et al., 2018). The observed pattern indicates a major contribution from the historical electrochemical fluorination (ECF) manufacturing process by the $3 \mathrm{M}$ company, typically yielding $30 \%$ branched isomers (Benskin et al., 2010). ECF PFOS from major Chinese producers has been reported to contain a similar percentage of branched PFOS (28-34\%) (Jiang et al., 2015). The second major synthesis route is telomerization based on linear telogens as the starting material, yielding primarily or exclusively linear isomers (Buck et al., 2011). Consequently, the significantly higher proportion of $b r$-PFOS in the Chinese Xi River $(77 \pm 8 \%)$ than in the other rivers (two-sample $t$-test, $\alpha=0.05, p<0.001$ ) is not explicable by these two synthesis routes. The differential transport of linear and branched isomers with a higher sorption of linear isomers to sediments can be a possible explanation for an enrichment of branched compounds in the water phase (Schulz et al., 2020).
However, the proportion of $b r$-PFOS in the Xi River sample FX_002, taken close to the wastewater outlet of the point source, is already $80 \%$ and there is no significant decrease in the proportion of linear isomers in the samples taken further downstream. The high proportion of $b r$-PFOS could be explained by degradation of branched PFOS precursors during the wastewater treatment and/or a synthesis route other than ECF or (linear) telomerization. Although commercial production has not been confirmed so far, telomerization employing a branched telogen as starting material has been described in patents to produce branched isomers (Grottenmüller et al., 2003; Millauer, 1971). For PFHxS, a similar difference between the Xi River and the German rivers was observed. In the Yangtze River, the contribution of $21 \pm 5 \%$ br-PFHxS was more similar to the German samples compared to a share of $43 \pm 12 \%$ brPFOS (Fig. 4A). This indicates that PFHxS and PFOS in the Yangtze River result from different types of production. 


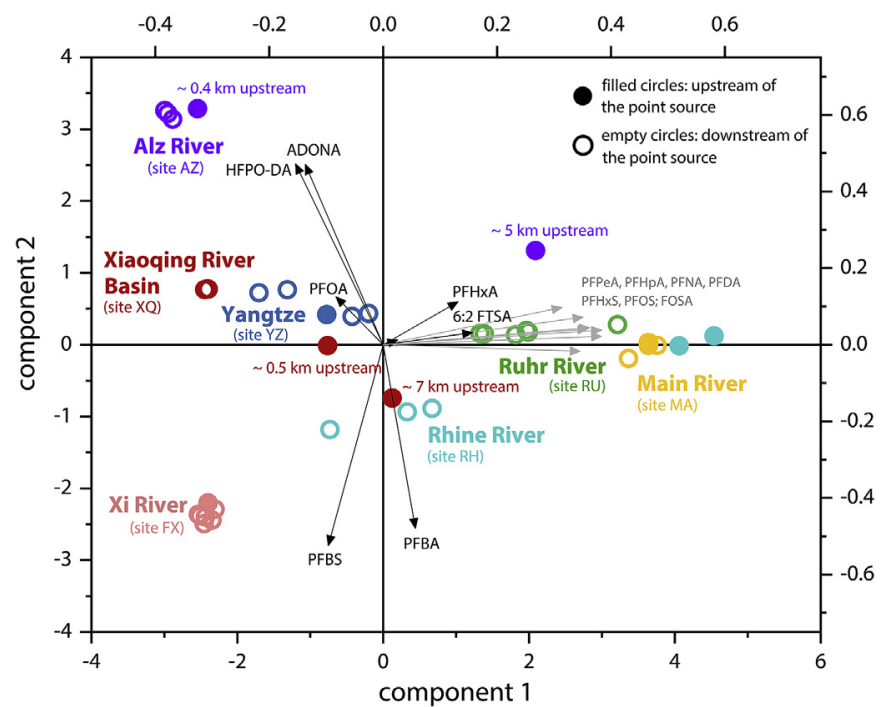

Fig. 3. Results from principal component analysis (PCA) on PFASs measured up- and downstream of the suspected point sources. The figure depicts only samples taken from the rivers at which the point sources were located, not including tributaries. The first and second components were plotted against each other and colored according to the point source. Dominant compounds for each component are shown as vectors.

For PFHpA and PFOA, the percentage of branched isomers was significantly higher in the Xiaoqing River Basin and in the Xi River Basin than in the German rivers (two-sample $t$-test, $\alpha=0.05$, $p<0.001$ ) (Fig. 4B). The percentage of branched isomers in commercial ECF PFOA products from Chinese manufacturers (20-26\%) has been reported to be similar to that of 3M ECF PFOA (22\%) (Jiang et al., 2015; Shi et al., 2015). br-PFOA in the Xiaoqing River Basin $(19 \pm 3 \%)$ and Xi River Basin $(20 \pm 2 \%)$ was comparable to that of the commercial ECF products, indicating that ECF is the dominant source. The lower percentage of $b r$-PFOA in other rivers, e.g. the Rhine River $(9.4 \pm 0.6 \%)$ and Main River $(8.7 \pm 0.5 \%)$, can likely be attributed to production of PFOA or fluorotelomer-based precursors by telomerization, yielding linear PFOA and diluting the ECF signature, or to different environmental fate processes.

A higher proportion of branched isomers in the Chinese environment compared to earlier studies focusing on Europe and North America (Benskin et al., 2010) has also been reported previously. This includes PFOA close to fluoropolymer manufacturing plants in the Xiaoqing River Basin (23\% branched) (Shi et al., 2015) and in the upper Yangtze River ( $26 \pm 5 \%$ branched) (Fang et al., 2020). These findings underline that the individual consideration of both linear and branched isomers is important to assess environmental and human health risks.

\subsection{Contributions of unknown precursors}

Molar conversion yields for oxidation tests with model precursors (section 2.6) were in good agreement with literature data (Houtz and Sedlak, 2012; Janda, 2019; Martin et al., 2019) (Table S13). Upon oxidation of $n: 2$ fluorotelomer sulfonic acids, a mix of PFCAs resulted, whereas PFOA was the prevailing product upon oxidation of sulfonamide-containing precursors. In contrast to the results of Houtz and Sedlak (2012), the sulfonamidecontaining precursors showed reproducible formation of $3-4 \%$ PFHpA in addition to the major product PFOA. This has also been reported by Martin et al. (2019). As its predecessor PFOA, the perfluorinated ether-based replacement compound HFPO-DA was stable under TOP assay conditions. This was also observed by Zhang et al. (2019) and indicates that the introduction of an ether bond
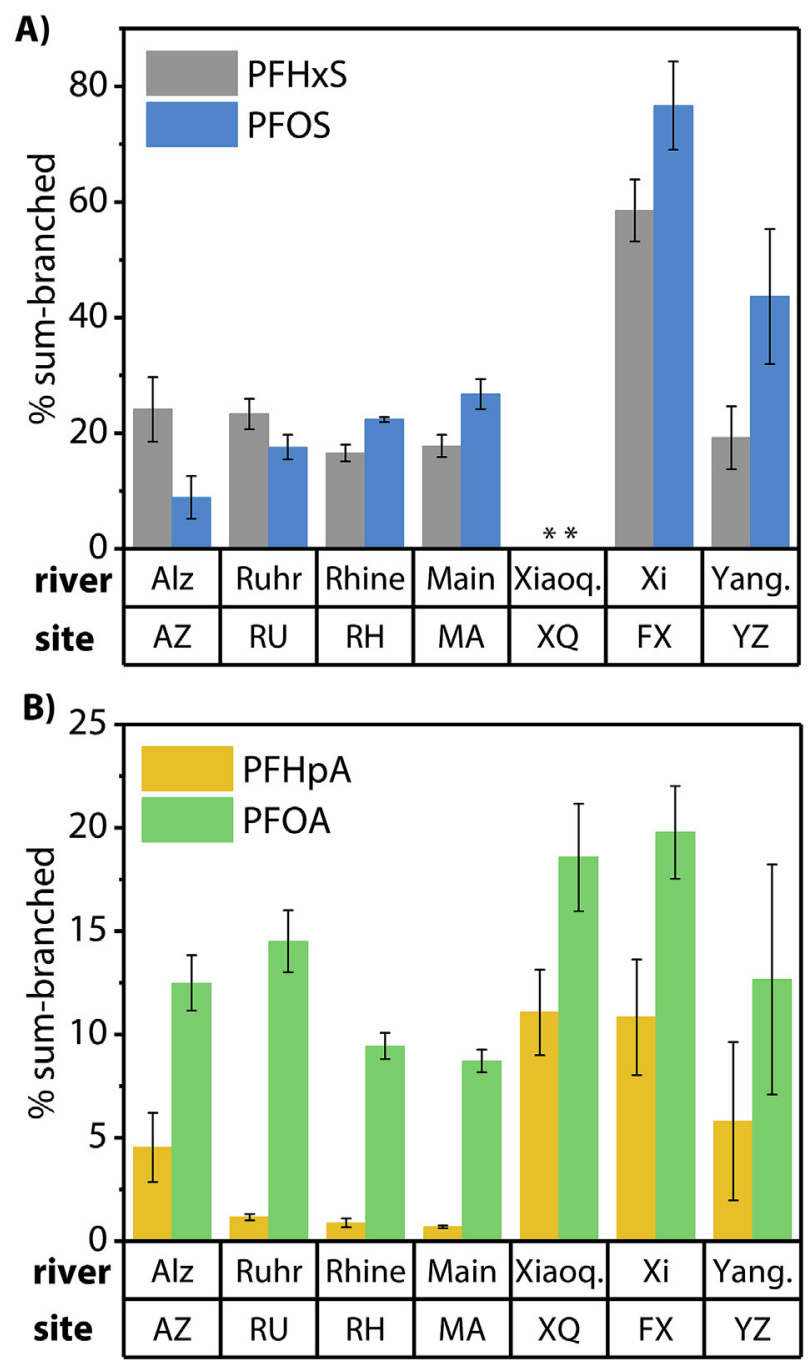

Fig. 4. Percent contribution of the sum of branched isomers A) to total PFHxS and PFOS, and B) to total PFHpA and PFOA in investigated rivers (mean \pm standard deviation [SD]). **As the results of both linear and branched isomers were below MDL in more than $50 \%$ of the samples, mean values were not calculated for the Xiaoqing River Basin (Xiaoq.) in A).

does not improve degradability and the compound represents a new terminal end product, which should be monitored when performing the TOP assay. In contrast, the polyfluorinated ether-based replacement compounds ADONA and 6:2 Cl-PFESA were partially degraded by $78 \pm 4 \%$ and $26 \pm 7 \%$ (Fig. S4). Based on the targeted PFASs, the oxidation products could not be identified. Zhang et al. (2019) reported perfluoro-3-methoxypropanoic acid (PFMOPrA) as oxidation product of ADONA, suggesting that the -O-CHFmoiety is the site of hydroxyl radical attack. In contrast to our observations, 6:2 Cl-PFESA was reported not degradable under TOP assay conditions by Zhang et al. (2019). Possible explanations for the different observations are different spiking levels and concentrations of added persulfate. However, in a mechanochemical destruction study, 6:2 Cl-PFESA also showed better degradability compared to the perfluorinated compound PFOS by milling with $\mathrm{KOH}$. Based on observations for additional compounds in their study, the authors concluded that the replacement of one fluorine atom by chlorine rather than the introduction of the ether bond improves the degradability of the molecule (Zhang et al., 2016).

Analysis of the river water samples resulted in an increase of $\Sigma \mathrm{C}_{4}-\mathrm{C}_{7}$ PFCAs by $18 \%-82 \%$ in German samples (mean $\pm \mathrm{SD}$ 
$59 \pm 19 \%$ ) and up to $32 \%$ in Chinese samples (15 $\pm 10 \%$ ), varying between countries, sources, and individual samples (Fig. 5). In general, a significantly higher increase of PFBA could be observed in German samples $(88 \pm 30 \%)$ than in Chinese samples $(12 \pm 14 \%)$ (two-sample $t$-test, $\alpha=0.05, p<0.001$ ). In contrast, PFHpA showed a significantly larger increase in Chinese samples $(21 \pm 15 \%)$ than in German samples $(2 \pm 9 \%)$ (two-sample $t$-test, $\alpha=0.05, p<0.001)$. This indicates the presence of a higher proportion of precursors to the short-chain compound PFBA in German rivers and precursors to the longer-chain compound PFHpA in Chinese rivers, reflecting the geographical shift of production.

The increase of PFOA was $<25 \%$ in Chinese samples, except for sample YZ_006 with a PFOA increase of 460\% (Table S22). This sample was not collected from flowing river water, but from stagnant, shallow water at the riverside. The large difference between the oxidized and unoxidized aliquot of this sample is possibly related to a higher impact of stack emissions of the nearby fluorochemical industrial park, which may contain unmonitored precursors of long-chain PFCAs, such as fluorotelomer alcohols (FTOHs) or polyfluoroalkyl phosphate esters (PAPs). Due to the differences between MQLs of oxidized and unoxidized samples (see section 2.6 and Table S14), $\mathrm{C}_{8}$ to $\mathrm{C}_{10}$ PFCAs were below MQL in at least one of the sample aliquots in more than $80 \%$ of the German samples. Consequently, the data were not considered for further evaluation. Results for individual samples are provided in Tables S21 and S22.

Amongst the precursors, 6:2 FTSA was detected in 30\% of the unoxidized samples, whereas it was not detectable after oxidation. Based on the molar conversion yields of 6:2 FTSA determined during validation of the TOP assay, the percentage of increase of $C_{4}$ to $C_{7}$ PFCAs that can be attributed to oxidation of 6:2 FTSA was calculated (Tables S23 and S24). In the eleven German samples, in which 6:2 FTSA was detected, 97-99\% of the molar increase of $C_{4}$ to $\mathrm{C}_{7}$ PFCAs remained unexplained (Fig. S6), pointing to the presence of unmonitored precursors. In the two Yangtze River samples YZ_003 and YZ_004, a comparatively high proportion of $83 \%$ and $29 \%$ of the molar increase of $C_{4}$ to $C_{7}$ PFCAs could be attributed to 6:2 FTSA, pointing to direct emissions of this precursor at the manufacturing site.

As observed in the oxidation tests with model substances, HFPO-DA was not degraded upon oxidation in the river water samples. In contrast, 6:2 Cl-PFESA was degraded partially in the samples (by $12 \pm 16 \%$ ) and ADONA was nearly fully oxidized, e.g., the ADONA concentration in the Alz River samples decreased from $740 \pm 43 \mathrm{ng} / \mathrm{L}$ pre-TOP to $1.8 \pm 1.0 \mathrm{ng} / \mathrm{L}$ post-TOP. As shown during method validation, the oxidation products of 6:2 Cl-PFESA and ADONA are not targeted. It can be assumed that the samples contain many additional PFASs that are missed by both target analysis and TOP assay. This includes non-targeted non-oxidizable PFASs and non-targeted PFASs that are oxidizable but degraded to non-targeted oxidation products. The inclusion of oxidation products other than PFCAs and of newly identified terminal products such as HFPO-DA can help in capturing a larger amount of total PFASs at a specific site.

\subsection{PFAS mass flow estimates}

PFAS mass flow estimates were calculated to draw comparisons to earlier studies and to provide first estimates for emerging compounds. The mean estimates for the sum of PFASs varied between $0.03 \mathrm{t} / \mathrm{y}$ for the Lahn River as a small River Rhine tributary and $100 \mathrm{t} / \mathrm{y}$ for the Yangtze River (mean annual discharge $41 \mathrm{~m}^{3} / \mathrm{s}$ versus $27,400 \mathrm{~m}^{3} / \mathrm{s}$ ). Estimates for the individual PFASs in the investigated rivers are provided in Table S25. It must be noted that this calculation is based on a small number of samples for each river, collected close to point sources at different distance to the river mouths at one point in time (Fig. S1). Mass flows may vary due to discontinuous emissions of PFASs by the industrial plants, variations in water discharge, or seasonal trends. Consequently, the provided PFAS mass flows must be considered as rough estimates.

The sum of the mass flows was highest for PFOA, with major contributions from the Chinese Xiaoqing River (mean $20 \mathrm{t} / \mathrm{y}$ ) and Yangtze River ( $43 \mathrm{t} / \mathrm{y}$ ). This is approximately 250 times higher than in the German Rhine River, a major contributor to PFOA discharges from the European continent (McLachlan et al., 2007; Lindim et al., 2016), underlining the significance of Chinese point sources for global PFOA emissions. PFOA mass flows were in the same order of magnitude as calculated in earlier studies for the Xiaoqing River (23-67 t/y) (Shi et al., 2015) and for the Yangtze River (6.7-26 t/y) (Wang et al., 2016a). However, the mass flow of PFHxA estimated for the Yangtze River in this study (41 t/y), based on samples from 2018, was considerably higher than estimated total PFHxA discharges from 19 Chinese rivers in 2013, including the Yangtze River (2.2-4.0 t/y) (Wang et al., 2016a). This finding suggests an increasing relevance of PFHxA in China.

Compared to PFOA and PFHxA, the mass flows of the short-chain compound PFBS in the German Rhine River had a higher contribution to the sum of transported PFASs. Although the mean annual discharge of the Rhine River is only approximately $6 \%$ of that of the Yangtze River, the PFBS mass flow in the Rhine River (2.3 t/y) was

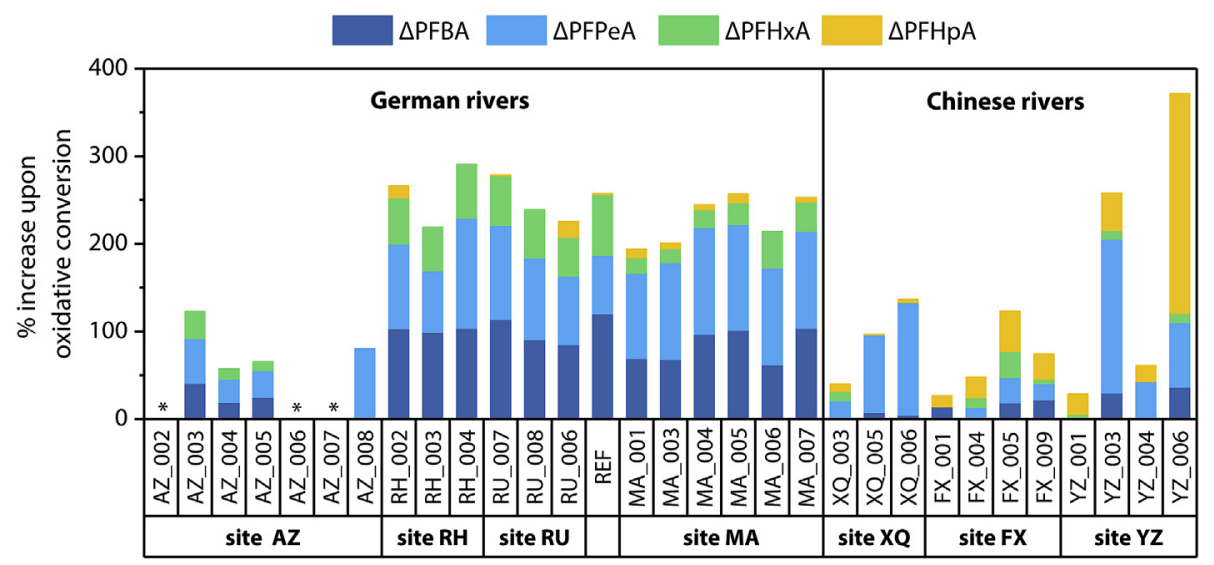

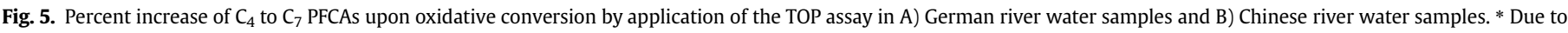
values $<\mathrm{MDL}$, the \% increase was not calculated for three samples from the Alz River. 
approximately half of that in the Yangtze River (4.3 t/y) (Wang et al., 2016a). Möller et al. (2010) estimated a PFBS mass flow of $5.1 \mathrm{t} / \mathrm{y}$ for the Rhine River based on samples taken at similar sampling locations in 2008. This indicates ongoing emissions from the point source at site RH for more than ten years. The comparatively high PFAS load in the Lower Rhine is underlined by monthly measurements by the State Agency for Nature, Environment and Consumer Protection (LANUV) of North Rhine-Westphalia, regularly reporting PFBS concentrations above the MQL of $10 \mathrm{ng} / \mathrm{L}$ for the metering points 000309 and 000504 since 2007 (LANUV, 2020).

With respect to the ether-based PFASs, mass flows of HFPO-DA were in the same range in the Yangtze River, Xiaoqing River, and the Alz River (0.9, 0.3 and $0.4 \mathrm{t} / \mathrm{yr}$, respectively), whereas the Alz River was the dominant contributor for ADONA ( $0.4 \mathrm{t} / \mathrm{y})$ and the Yangtze River for 6:2 Cl-PFESA (0.2 t/y).

The increase of C4-C7 PFCAs upon oxidative conversion in the TOP assay (Fig. 5) indicates that riverine discharge derived from conventional target analysis reveals only part of the PFASs present in a sample. This can ultimately be transformed to endpoint PFASs of concern, e.g. when transported from rivers to the seas and further to remote areas.

\section{Conclusions}

The analysis of 29 legacy and emerging PFASs in German and Chinese river water impacted by industrial point sources revealed source- and country-specific PFAS fingerprints. The estimation of annual riverine mass flows underlined the ongoing high emissions of the legacy compound PFOA in China from point sources in the Xiaoqing River Basin and along the Yangtze River. HFPO-DA, as one of its replacement compounds in fluoropolymer production, was detected in $98 \%$ of the samples in this study, with the highest concentration found in the vicinity of a German fluoropolymer manufacturing site. As PFOA has been recently added to the Stockholm Convention (BRS Secretariat, 2019), it can be expected that major producers in continental Asia will reduce emissions of PFOA and its precursors in the foreseeable future (Wang et al., 2017). Although HFPO-DA is not as well-studied as PFOA, several studies indicate that it has similar properties and can be considered as a "regrettable substitute" to PFOA (Gomis et al., 2015; Wang et al., 2015; Gomis et al., 2018). In order to avoid repeating the industrial transition from PFOA to HFPO-DA in continental Asia, which has been taking place in Western countries since the 2000s, the evaluation and regulation of HFPO-DA and other replacements on an international level is essential.

Both the higher percentage of branched isomers at Chinese sites compared to German rivers and the increase of $C_{4}$ to $C_{7}$ PFCAs upon oxidative conversion in the TOP assay underline the limitations of conventional target analysis. Dependent on the type of point source and the spectrum of target analytes, human and environmental health risks may be considerably underestimated, when analyzing only a small proportion of the PFASs on the worldwide market. Although the TOP assay is a more inclusive method, it still depends on targeted PFASs and only provides a minimum estimate of the unknown oxidizable precursors present in an environmental sample. Consequently, unknown PFASs in the samples can be missed by both target analysis and the TOP assay. To address this gap, the results from target analysis and the TOP assay will be complemented by a follow-up study (Joerss et al., 2020; in preparation) using an HRMS-based approach.

\section{Contribution of the authors}

Hanna Joerss: Conceptualization; Investigation; Methodology; Roles/Writing - original draft; Thekla-Regine Schramm:
Investigation; Methodology; Linting Sun: Investigation; Chao Guo: Investigation; Jianhui Tang: Conceptualization; Funding acquisition; Project administration; Resources; Supervision; Writing review \& editing, Ralf Ebinghaus: Funding acquisition; Project administration; Resources; Supervision; Writing - review \& editing.

\section{Declaration of competing interest}

The authors declare that they have no known competing financial interests or personal relationships that could have appeared to influence the work reported in this paper.

\section{Acknowledgements}

We thank Yu Jing for his help during the sampling campaign in China. This study was supported by the SOA (State Oceanic Administration of China)-BMBF (German Federal Ministry of Education and Research) joint project MEGAPOL [03F0786C], the National Natural Science Foundation of China [U1806207], and the Key Deployment Project of the Centre for Ocean Mega-Science, Chinese Academy of Sciences [COMS2019J08].

\section{Appendix A. Supplementary data}

Supplementary data to this article can be found online at https://doi.org/10.1016/j.envpol.2020.115567.

\section{References}

Bao, J., Liu, W., Liu, L., Jin, Y., Dai, J., Ran, X., Zhang, Z., Tsuda, S., 2011. Perfluorinated compounds in the environment and the blood of residents living near fluorochemical plants in fuxin, China. Environ. Sci. Technol. 45 (19), 8075-8080.

Benskin, J.P., de Silva, A.O., Martin, J.W., 2010. Isomer profiling of perfluorinated substances as a tool for source tracking: a review of early findings and future applications. In: Perfluorinated Alkylated Substances. Reviews of Environmental Contamination and Toxicology, Volume 208. Springer, New York pp. $111-160$.

Bode, H., 1998. Control of heavy metal emission from metal plating industry in a German river basin. Water Sci. Technol. 38 (4), 121-129.

Boiteux, V., Dauchy, X., Bach, C., Colin, A., Hemard, J., Sagres, V., Rosin, C., Munoz, J.F. 2017. Concentrations and patterns of perfluoroalkyl and polyfluoroalkyl substances in a river and three drinking water treatment plants near and far from a major production source. Sci. Total Environ. 583, 393-400.

Brs Secretariat Secretariat of the Basel Rotterdam and Stockholm Conventions 2019. Listing of Perfluorooctanoic Acid (PFOA), its Salts and PFOA-Related Compounds. UNEP/POPS/COP.9-SC-9/12. http://chm.pops.int/TheConvention/ ConferenceoftheParties/Meetings/COP9/tabid/7521/Default.aspx. (Accessed 1 November 2019).

Buck, R.C., Franklin, J., Berger, U., Conder, J.M., Cousins, I.T., de Voogt, P., Jensen, A.A Kannan, K., Mabury, S.A., van Leeuwen, S.P., 2011. Perfluoroalkyl and polyfluoroalkyl substances in the environment: terminology, classification, and origins. Integrated Environ. Assess. Manag. 7 (4), 513-541.

Chandramouli, B., Benskin, J.P., Hamilton, M.C., Cosgrove, J.R., 2015. Sorption of perand polyfluoroalkyl substances (PFASs) on filter media: implications for phase partitioning studies. Environ. Toxicol. Chem. 34 (1), 30-36.

Chen, H., Yao, Y., Zhao, Z., Wang, Y., Wang, Q., Ren, C., Wang, B., Sun, H., Alder, A.C., Kannan, K., 2018. Multimedia distribution and transfer of per- and polyfluoroalkyl substances (PFASs) surrounding two fluorochemical manufacturing facilities in fuxin, China. Environ. Sci. Technol. 52 (15), 8263-8271.

Fang, S., Sha, B., Yin, H., Bian, Y., Yuan, B., Cousins, I.T., 2020. Environment occurrence of perfluoroalkyl acids and associated human health risks near a major fluorochemical manufacturing park in southwest of China. J. Hazard Mater. 396 122617.

Fromme, H., Wöckner, M., Roscher, E., Völkel, W., 2017. ADONA and perfluoroalkylated substances in plasma samples of German blood donors living in South Germany. Int. J. Hyg. Environ. Health 220 (2), 455-460. Part B.

Gobelius, L., Hedlund, J., Dürig, W., Tröger, R., Lilja, K., Wiberg, K., Ahrens, L., 2018 Per- and polyfluoroalkyl substances in Swedish groundwater and surface water: implications for environmental quality standards and drinking water guidelines. Environ. Sci. Technol. 52 (7), 4340-4349.

Gomis, M.I., Wang, Z., Scheringer, M., Cousins, I.T., 2015. A modeling assessment of the physicochemical properties and environmental fate of emerging and nove per- and polyfluoroalkyl substances. Sci. Total Environ. 505, 981-991.

Gomis, M.I., Vestergren, R., Borg, D., Cousins, I.T., 2018. Comparing the toxic potency in vivo of long-chain perfluoroalkyl acids and fluorinated alternatives. Environ. Int. 113, 1-9. 
Grottenmüller, R., Knaup, W., Probst, A., Dullinger, B., 2003. Process for the preparation of perfluorocarboxylic acids. US Patent 6, 515, 172 B2. Clariant.

Heydebreck, F., Tang, J., Xie, Z., Ebinghaus, R., 2015. Alternative and legacy perfluoroalkyl substances: differences between European and Chinese river/estuary systems. Environ. Sci. Technol. 49 (14), 8386-8395.

Houtz, E.F., Sedlak, D.L., 2012. Oxidative conversion as a means of detecting precursors to perfluoroalkyl acids in urban runoff. Environ. Sci. Technol. 46 (17), 9342-9349.

Janda, J., 2019. Polare Perfluoralkylcarbonsäuren - Bestimmung in aquatischen Proben und Untersuchungen zu ihren Präkursoren in Wasser und Feststoffen (in German). In: Dissertation. Springer Spektrum, Heidelberg.

Jiang, W., Zhang, Y., Yang, L., Chu, X., Zhu, L., 2015. Perfluoroalkyl acids (PFAAs) with isomer analysis in the commercial PFOS and PFOA products in China. Chemosphere 127, 180-187.

Jin, H., Zhang, Y., Zhu, L., Martin, J.W., 2015. Isomer profiles of perfluoroalkyl substances in water and soil surrounding a Chinese fluorochemical manufacturing park. Environ. Sci. Technol. 49 (8), 4946-4954.

in preparation Joerss, H., Menger, F., Tang, J., Ebinghaus, R., Ahrens, L., 2020. Discovery of Emerging and Novel Per- and Polyfluoroalkyl Substances in Chinese and German River Water Using a Suspect Screening Approach.

Joerss, H., Apel, C., Ebinghaus, R., 2019. Emerging per- and polyfluoroalkyl substances (PFASs) in surface water and sediment of the North and Baltic Seas. Sci. Total Environ. 686, 360-369.

Kissa, E., 2001. Fluorinated Surfactants and Repellents, second ed. Marcel Dekker New York.

LANUV, State Agency for Nature, Environment and Consumer Protection of North Rhine-Westphalia, 2020. PFT-Werte an Oberflächengewässern (in German) https://www.elwasweb.nrw.de/elwas-hygrisc/gues/pft_ow.php?exhibit-uselocal-resources\#2. (Accessed 20 August 2020).

Lindim, C., van Gils, J., Cousins, I.T., 2016. Europe-wide estuarine export and surface water concentrations of PFOS and PFOA. Water Res. 103, 124-132.

Liu, Y., D’Agostino, L., Qu, G., Jiang, G., Martin, J.W., 2019. High-resolution mass spectrometry (HRMS) methods for nontarget discovery and characterization of poly- and perfluoroalkyl substances (PFASs) in environmental and human samples. Trends Anal. Chem. 121, 115420.

Martin, D., Munoz, G., Mejia-Avendaño, S., Duy, S.V., Yao, Y., Volchek, K., Brown, C.E., Liu, J., Sauvé, S., 2019. Zwitterionic, cationic, and anionic perfluoroalkyl and polyfluoroalkyl substances integrated into total oxidizable precursor assay of contaminated groundwater. Talanta 195, 533-542.

McDonough, C.A., Guelfo, J.L., Higgins, C.P., 2019. Measuring total PFASs in water: the tradeoff between selectivity and inclusivity. Curr. Opin. Environ. Sci. Health 7, $13-18$

McLachlan, M.S., Holmstrom, K.E., Reth, M., Berger, U., 2007. Riverine discharge of perfluorinated carboxylates from the European continent. Environ. Sci. Technol. 41 (21), 7260-7265.

Millauer, H., 1971. Process for the preparation of pentafluoroethyl iodide and heptafluoroisopropyl iodide. Washington (DC): US Patent Office. Patent 3, 829, 512. Hoechst.

Möller, A., Ahrens, L., Sturm, R., Westerveld, J., van der Wielen, F., Ebinghaus, R., de Voogt, P., 2010. Distribution and sources of polyfluoroalkyl substances (PFAS) in the River Rhine watershed. Environ. Pollut. 158 (10), 3243-3250.

Norwegian Environment Agency, 2017. Sources of Perfluorobutane Sulfonic Acid (PFBS) in the Environment. Report M-759/2017. https://www.miljodirektoratet no/globalassets/publikasjoner/M759/M759.pdf. (Accessed 19 April 2020).

OECD; Organisation for Economic Co-operation and Development, 2018. Toward a new comprehensive global database of per- and polyfluoroalkyl substances
(PFASs): summary report on updating the OECD 2007 list of per- and polyfluoroalkyl substances (PFASs). Series on Risk Management 39, 1-24.

Pan, Y., Zhang, H., Cui, Q., Sheng, N., Yeung, L.W.Y., Sun, Y., Guo, Y., Dai, J., 2018. Worldwide distribution of novel perfluoroether carboxylic and sulfonic acids in surface water. Environ. Sci. Technol. 52 (14), 7621-7629.

Schulz, K., Silva, M.R., Klaper, R., 2020. Distribution and effects of branched versus linear isomers of PFOA, PFOS, and PFHxS: a review of recent literature. Sci. Total Environ. 733, 139186.

Shi, Y., Vestergren, R., Xu, L., Song, X., Niu, X., Zhang, C., Cai, Y., 2015. Characterizing direct emissions of perfluoroalkyl substances from ongoing fluoropolymer production sources: a spatial trend study of Xiaoqing River, China. Environ. Pollut. 206, 104-112.

Skutlarek, D., Exner, M., Farber, H., 2006. Perfluorinated surfactants in surface and drinking waters. Environ. Sci. Pollut. Res. Int. 13 (5), 299-307.

Song, X., Vestergren, R., Shi, Y., Huang, J., Cai, Y., 2018. Emissions, transport, and fate of emerging per- and polyfluoroalkyl substances from one of the major fluoropolymer manufacturing facilities in China. Environ. Sci. Technol. 52 (17), 9694-9703.

Wang, S., Huang, J., Yang, Y., Hui, Y., Ge, Y., Larssen, T., Harman, C., 2013a. First report of a Chinese PFOS alternative overlooked for 30 years: its toxicity, persistence, and presence in the environment. Environ. Sci. Technol. 47 (18), 10163-10170.

Wang, Z., Cousins, I.T., Scheringer, M., Hungerbühler, K., 2013b. Fluorinated alternatives to long-chain perfluoroalkyl carboxylic acids (PFCAs), perfluoroalkane sulfonic acids (PFSAs) and their potential precursors. Environ. Int. 60, 242-248.

Wang, Z., Cousins, I.T., Scheringer, M., Buck, R.C., Hungerbühler, K., 2014. Global emission inventories for C4-C14 perfluoroalkyl carboxylic acid (PFCA) homologues from 1951 to 2030, Part I: production and emissions from quantifiable sources. Environ. Int. 70, 62-75.

Wang, Z., Cousins, I.T., Scheringer, M., Hungerbuehler, K., 2015. Hazard assessment of fluorinated alternatives to long-chain perfluoroalkyl acids (PFAAs) and their precursors: status quo, ongoing challenges and possible solutions. Environ. Int. $75,172-179$.

Wang, T., Vestergren, R., Herzke, D., Yu, J., Cousins, I.T., 2016a. Levels, isomer profiles, and estimated riverine mass discharges of perfluoroalkyl acids and fluorinated alternatives at the mouths of Chinese rivers. Environ. Sci. Technol. 50 (21), 11584-11592.

US EPA, 2020. United States Environmental Protection Agency. PFAS master list of PFAS substances. https://comptox.epa.gov/dashboard/chemical_lists/ pfasmaster. (Accessed 14 August 2020).

Wang, Z., Cousins, I.T., Berger, U., Hungerbühler, K., Scheringer, M., 2016b. Comparative assessment of the environmental hazards of and exposure to perfluoroalkyl phosphonic and phosphinic acids (PFPAs and PFPiAs): current knowledge, gaps, challenges and research needs. Environ. Int. 89-90, 235-247.

Wang, Z., DeWitt, J.C., Higgins, C.P., Cousins, I.T., 2017. A never-ending story of perand polyfluoroalkyl substances (PFASs)? Environ. Sci. Technol. 51 (5), 2508-2518.

Ye, F., Tokumura, M., Islam, M.S., Zushi, Y., Oh, J., Masunaga, S., 2014. Spatial distribution and importance of potential perfluoroalkyl acid precursors in urban rivers and sewage treatment plant effluent - case study of Tama River, Japan. Water Res. 67, 77-85

Zhang, K., Cao, Z., Huang, J., Deng, S., Wang, B., Yu, G., 2016. Mechanochemical destruction of Chinese PFOS alternative F-53B. Chem. Eng. J. 286, 387-393.

Zhang, C., Hopkins, Z.R., McCord, J., Strynar, M.J., Knappe, D.R.U., 2019. Fate of perand polyfluoroalkyl ether acids in the total oxidizable precursor assay and implications for the analysis of impacted water. Environ. Sci. Technol. Lett. 6 (11), 662-668. 